\title{
Unified no-scale model of modulus fixing, inflation, supersymmetry breaking, and dark energy
}

\author{
John Ellis, ${ }^{1}$ Dimitri V. Nanopoulos, ${ }^{2}$ Keith A. Olive, ${ }^{3}$ and Sarunas Verner ${ }^{3}$ \\ ${ }^{1}$ Theoretical Particle Physics and Cosmology Group, Department of Physics, King's College London, \\ London WC2R 2LS, United Kingdom, Theoretical Physics Department, \\ CERN, CH-1211 Geneva 23, Switzerland, and NICPB, Rävala pst. 10, 10143 Tallinn, Estonia \\ ${ }^{2}$ George P. and Cynthia W. Mitchell Institute for Fundamental Physics and Astronomy, \\ Texas A\&M University, College Station, Texas 77843, USA, \\ Astroparticle Physics Group, Houston Advanced Research Center (HARC), Mitchell Campus, \\ Woodlands, Texas 77381, USA, and Academy of Athens, Division of Natural Sciences, \\ Athens 10679, Greece \\ ${ }^{3}$ William I. Fine Theoretical Physics Institute, School of Physics and Astronomy, University of Minnesota, \\ Minneapolis, Minnesota 55455, USA
}

(Received 25 March 2019; published 18 July 2019)

\begin{abstract}
We present a minimal $\mathrm{SU}(2,1) / \mathrm{SU}(2) \times \mathrm{U}(1)$ no-scale model that unifies modulus fixing, Starobinskylike inflation, an adjustable scale for supersymmetry breaking, and the possibility of a small cosmological constant, a.k.a. dark energy.
\end{abstract}

DOI: 10.1103/PhysRevD.100.025009

\section{INTRODUCTION}

Physics contains many hierarchies of mass scales, starting from the Planck scale $M_{P} \sim 10^{19} \mathrm{GeV}$ at which the effects of quantum gravity must become important, through the energy scale of cosmological inflation, which is $\lesssim 10^{13} \mathrm{GeV}$, through the electroweak scale $\sim 100 \mathrm{GeV}$, down to the energy scale of dark energy, a.k.a. the cosmological constant, which is $\sim 10^{-3} \mathrm{eV}$. What are the origins of these hierarchies, and how can they be stabilized in a natural way despite the depredations of quantum corrections? Diverse origins have been proposed, and this paper does not claim any progress in elucidating this aspect of the hierarchies. Instead, we focus on the question of how they can be accommodated within a simple framework that incorporates a mechanism for stabilizing hierarchies of mass scales.

That framework is provided by supersymmetry, which could stabilize the electroweak hierarchy if the supersymmetry-breaking scale is $\lesssim 1 \mathrm{TeV}$ [1], and could also stabilize the parameters of an inflationary scalar potential at some scale $\ll M_{P}$ [2]. On the other hand, simple supersymmetry is insufficient by itself to render natural the small magnitude of the cosmological constant, and it would need to be supplemented by dynamical mechanisms

Published by the American Physical Society under the terms of the Creative Commons Attribution 4.0 International license. Further distribution of this work must maintain attribution to the author(s) and the published article's title, journal citation, and DOI. Funded by SCOAP. to generate the hierarchies of mass scales. In the context of cosmology, supersymmetry must be combined with general relativity within some form of supergravity theory [3]. For this we advocate no-scale supergravity [4,5], since it does not have any of the deep $\mathcal{O}\left(M_{P}^{4}\right)$ anti-de Sitter "holes" in the effective potential that are endemic in other supergravity theories with matter fields. Moreover, no-scale supergravity appears generically in compactifications of string theory [6], which we regard as the most promising candidate for a quantum theory of gravity. One may anticipate that string theory is the UV completion of the model presented below, though our model does require specific knowledge of string theory other than this recognition that no-scale supergravitiy is a generic consequence of string models [6].

No-scale supergravity has been shown to yield Starobinsky-like models of inflation [7], under suitable conditions on the theoretical parameters [8], and we have recently characterized general conditions under which de Sitter (dS) vacua can be accommodated within no-scale supergravity [9]. Upgrading such models to something resembling the Standard Model (SM) in a more realistic way requires a deeper discussion on how matter fields should be incorporated, that should also include a mechanism for supersymmetry breaking. Previously, this has often been done by invoking some variant of the Polonyi model in which supersymmetry breaking originates dynamically within a hidden sector [10-14].

In this paper we build upon $[9,15]$ generalizing the characterization of dS no-scale supergravity models with $\mathrm{SU}(2,1) / \mathrm{SU}(2) \times \mathrm{U}(1)$ Kähler manifolds. Without extending the field content, we introduce a mechanism that allows 
for a transition between two dS vacua, one that can accommodate Starobinsky-like inflation and one with an amount of vacuum energy that could be very small, like the present cosmological constant (dark energy), without invoking any external mechanism such as uplifting by fibers [16]. As we show, this class of models also allows for supersymmetry breaking with a magnitude suitable for stabilizing the electroweak hierarchy, without invoking any hidden sector à la Polonyi. Additionally, a mechanism proposed previously $[17,18]$ can be used to fix the compactification modulus of the $\mathrm{SU}(2,1) / \mathrm{SU}(2) \times \mathrm{U}(1)$ model.

\section{A UNIFIED SU(2, 1)/SU(2) x U(1) NO-SCALE MODEL}

We recall that a supergravity theory is described by a Kähler function $G \equiv K+\ln W+\ln W^{\dagger}$, where $K$ is a Hermitian Kähler potential and $W$ is a holomorphic superpotential. The scalar kinetic terms of the Lagrangian are given by $\mathcal{L}_{k i n}=K^{i j^{*}} \partial_{\mu} \Phi_{i} \partial^{\mu} \Phi_{j}^{*}$, where $K^{i j^{*}} \equiv \partial^{2} K / \partial_{\mu} \Phi_{i} \partial^{\mu} \Phi_{j}^{*}$ is the Kähler metric, and the effective scalar potential is given by

$$
V=e^{G}\left[\frac{\partial G}{\partial \Phi_{i}} K_{i j^{*}} \frac{\partial G}{\partial \Phi_{j}^{*}}-3\right],
$$

where $K_{i j^{*}}$ is the inverse of the Kähler metric.

Our model is characterized by the following Kähler potential

$$
\begin{aligned}
K= & -3 \alpha \ln \left[T+T^{\dagger}-\frac{\phi \phi^{\dagger}}{3}-\frac{X^{i} X_{i}^{\dagger}}{3}\right. \\
& \left.+b\left(T+T^{\dagger}-2 d\right)^{4}+c\left(T-T^{\dagger}\right)^{4}\right],
\end{aligned}
$$

where $\alpha$ is a curvature parameter (hereafter we set $d=1 / 2$ for definiteness) and the superpotential $W$ can be written as follows:

$$
W=W_{I}(T, \phi)+W_{\mathrm{SM}}(X, \phi)+W_{d S}(T, \phi),
$$

where $W_{I}$ is responsible for inflation, $W_{\mathrm{SM}}$ contains Standard Model interactions (possibly with the inflaton for reheating), and $W_{d s}$ will provide both supersymmetry breaking and dark energy. More specifically, these are

$$
\begin{aligned}
W_{I} & =M\left(\frac{\phi^{2}}{2}-\frac{\phi^{3}}{3 \sqrt{3}}\right), \\
W_{\mathrm{SM}} & =\mu X^{i} X^{j}+\lambda X^{i} X^{j} X^{k}+y \phi X^{i} X^{j},
\end{aligned}
$$

and

$$
W_{d S}=a_{1}\left(2 T-\frac{\phi^{2}}{3}\right)^{n_{-}}-a_{2}\left(2 T-\frac{\phi^{2}}{3}\right)^{n_{+}} .
$$

Equations (2)-(6) fully define our model. While inflation based on Eq. (4) was first introduced in [8], combining this with $W_{\text {SM }}$ and $W_{d S}$ in Eq. (3) is the key novel feature in the model considered below. In particular, adding $W_{d S}$ to $W_{I}$ preserves Starobinsky-like inflationary evolution while breaking supersymmetry and leaving residual vacuum energy suitable for dark energy today. This is accomplished in a remarkably simple form without the necessity of addition field content. We now preview the interpretations of these expressions, before discussing them in more detail in the bulk of the paper.

\section{SUPERSYMMETRY BREAKING AND THE COSMOLOGICAL CONSTANT}

The complex field $T$ can be interpreted as a volume modulus of compactification, and $\phi$ as another gaugesinglet modulus acting as the inflaton. Together they parametrize the no-scale $\mathrm{SU}(2,1) / \mathrm{SU}(2) \times \mathrm{U}(1)$ coset manifold [15,19], while $X^{i}$ represent gauge-non-singlet matter fields such as those appearing in the SM. The quartic terms in (2) fix $T[17,18], W_{I}$ in (4) fixes the inflaton $\phi$ and enables Starobinsky-like inflation with an energy scale $\mathcal{O}(M)$ (other forms for $W_{I}$ are also possible: see $[15,18]$ ), and $W_{\mathrm{SM}}$ in (5) contains bilinear and trilinear terms of the generic forms appearing in SM-like superpotentials as well as a coupling of the inflaton to matter to allow for reheating. The novel terms in (3) are those in (6) with coefficients $a_{1,2}$, which have functional forms that are holomorphic versions of the corresponding Hermitian terms of the gauge singlets in the Kähler potential (2). Taken alone, $W_{d S}$ leads to a de Sitter solution for all real values of $\phi$ and $T$. The couplings $a_{1}$ and $a_{2}$ determine the magnitudes of soft supersymmetry breaking and the cosmological constant, which are $\mathcal{O}\left(a_{1}-a_{2}\right)$ and $\mathcal{O}\left(a_{1} a_{2}\right)$, respectively. Choosing $a_{1}=\mathcal{O}\left(10^{-16}\right)$ and $a_{2} \ll$ $a_{1}$ (or vice versa) would yield a cosmological constant and soft supersymmetry breaking of the desired magnitudes. Noscale supergravity theories derived from string models have, in general, additional moduli such as the dilaton and complex structure moduli. For simplicity, we assume that these are already fixed at scales above the inflationary scale. Throughout, we work in units of the reduced Planck mass, $M_{P}=1 /\left(8 \pi G_{N}\right) \approx 2.4 \times 10^{18} \mathrm{GeV}$.

We consider now in more detail the dS/dark energy sector $W_{d S}$ (6). Constructions of dS vacua with multiple moduli in $\mathrm{SU}(1,1) / \mathrm{U}(1) \times \mathrm{U}(1)$ no-scale supergravity were discussed in [9], and can be extended to general $\mathrm{SU}(\mathrm{N}, 1) / \mathrm{SU}(\mathrm{N}) \times \mathrm{U}(1)$ Kähler coset manifolds via the Kähler potential $K=-3 \alpha \ln \left[T+T^{\dagger}-\frac{\phi_{i} \phi_{i}^{\dagger}}{3}\right]$. We find that dS vacua solutions can be obtained from a superpotential $W_{d S}$ of the form (6) with $\phi \rightarrow \phi_{i}$ and exponents given by $n_{ \pm}=\frac{3}{2}(\alpha \pm \sqrt{\alpha})[9,20,21]$. Holomorphy of the superpotential requires $\alpha \geq 1$, and the no-scale case $K$ corresponds to $\alpha=1$ [22]. We assume that the imaginary parts of the moduli fields are fixed to $\langle\operatorname{Im} T\rangle=0$ and $\left\langle\operatorname{Im} \phi_{i}\right\rangle=0$, which can be realized by introducing higher-order terms in the Kähler potential such as those in the second line 
of (2), as was shown in $[17,18]$, or (in some cases) by the dynamics of the potential. Specializing to the $\mathrm{SU}(2,1) /$ $\mathrm{SU}(2) \times \mathrm{U}(1)$ no-scale case and inserting the expressions (2) and (6) into (1), we find the following effective scalar potential at the minimum:

$$
V=12 a_{1} a_{2}
$$

for all values of $\operatorname{Re} T$ and $\operatorname{Re} \phi$, which corresponds to a de Sitter vacuum when $a_{1}$ and $a_{2}$ are of the same sign. Thus the dS/dark energy superpotential $W_{d S}$ yields a cosmological constant (7) following the end of inflation.

Combining $W_{d S}$ with a suitable inflationary superpotential $W_{I}$, we can incorporate soft supersymmetry breaking without adding an additional Polonyi sector $[10,11]$ or introducing other possible dynamics [16]. To this end, we consider an inflationary superpotential $W_{I}$ that vanishes when the complex scalar fields $T$ and $\phi_{i}$ are fixed at the potential minimum; i.e., we do not induce supersymmetry breaking through $W_{I}$, which typically has a mass scale of the order of the inflationary scale $\sim 10^{13} \mathrm{GeV}$. When the volume modulus $T$ is stabilized through the quartic terms in Eq. (2) so that $\operatorname{Re} T=1 / 2$ and $\operatorname{Im} T=0$, the inflaton is stabilized so that $\operatorname{Im} \phi=0$ throughout inflation and $\operatorname{Re} \phi=0$ at the end of inflation. Supersymmetry breaking is generated through an $F$ term for $T$, which is given (for $\alpha=1$ ) by

$$
\begin{aligned}
F_{T} & =-e^{G / 2} K_{i j^{*}} G^{j}=-m_{3 / 2}\left(K_{T}+W_{T} / W\right) / 3 \\
& =\left(a_{1}+a_{2}\right) \neq 0
\end{aligned}
$$

at the minimum, and is independent of the inflationary scale $M$. The gravitino mass is simply given by $m_{3 / 2}=$ $a_{1}-a_{2}$ [23].

Supersymmetry breaking with a Minkowski vacuum would be obtained if either $a_{1}$ or $a_{2}$ vanishes, but we are interested here in models with $a_{1,2} \neq 0$. Specifically, we choose $a_{1}-a_{2}=\mathcal{O}\left(10^{-16}\right)$ in order that the gravitino mass be $\mathcal{O}(1) \mathrm{TeV}$ [24]. If we also choose $a_{2}=\mathcal{O}\left(10^{-104}\right)$, we would obtain a value of the dark energy density (cosmological constant) comparable to the present value, $\mathcal{O}\left(10^{-120}\right)$. However, this is not the appropriate choice, since we expect other contributions to the present vacuum energy density, specifically from electroweak gauge symmetry breaking and confinement in QCD, which are estimated to be $\mathcal{O}\left(m_{W}^{4}\right) \sim$ $\mathcal{O}\left(10^{-68}\right)$ and $\mathcal{O}\left(\Lambda_{\mathrm{QCD}}^{4}\right) \sim \mathcal{O}\left(10^{-80}\right)$, respectively. These can be accommodated together with the present value of the dark energy density by choosing $a_{1}=\mathcal{O}\left(10^{-16}\right)$ and $a_{2}=$ $\mathcal{O}\left(10^{-52}\right)$ with the values finely tuned to cancel the electroweak and QCD contributions so that the net value of the dark energy is $\mathcal{O}\left(10^{-120}\right)$. It is also possible that $a_{2}=\mathcal{O}\left(10^{-20}\right)$ if the residual vacuum density is canceled by a grand unified theory (GUT) phase transition of order $m_{3 / 2}^{2} M_{\mathrm{GUT}}^{2}$ [25]. The couplings $a_{1}$ and $a_{2}$ are radiatively stable, but the required fine-tuning is inelegant. We have no suggestion on how it may be achieved dynamically in a natural way, but it does provide an effective framework for the different relevant mass scales without additional fields or resorting to uplifting with additional string dynamics.

\section{INFLATIONARY DYNAMICS}

One expects that the mass of the inflaton may be of order $M \sim \mathcal{O}\left(10^{-5}\right)$, in which case we can safely ignore the mixing terms between $a_{1,2}$ and $M$ during inflation because $a_{2} \ll a_{1} \ll M$, so the constants $a_{1,2}$ do not affect the slow-roll inflationary dynamics. As was shown in $[8,18]$, the Starobinsky inflationary potential can be recovered in noscale $\mathrm{SU}(2,1) / \mathrm{SU}(2) \times \mathrm{U}(1)$ supergravity from the WessZumino-like superpotential $W_{I}$ in (4), and we recall briefly some of the results. For simplicity, we consider the Kähler potential (2) with two moduli fields $\phi$ and $T$, and set the curvature parameter to $\alpha=1$. We assume that the scalar field $T$ is fixed by the quartic terms in Eq. (2), acquiring a VEV $\langle T\rangle=1 / 2$. The couplings $b$ and $c$ are expected to both be $\gg 1$, corresponding to inverse mass scales $b, c \propto 1 / \Lambda_{T}^{2}$, with $\Lambda_{T} \ll M_{P}$, similar to strong stabilization in some Polonyi models [12,13,26-32]. In the limit $a_{2} \rightarrow 0$, if $b=c$, the two real fields associated with $T$ acquire the same mass. However, in the absence of supersymmetry breaking both remain massless [18] and only acquire a mass when supersymmetry is broken, $m_{R e T}=4 \sqrt{3 b} a_{1} \simeq 4 \sqrt{3 b} m_{3 / 2}$, $m_{\text {ImT }}=4 \sqrt{3 c} a_{1} \simeq 4 \sqrt{3 c} m_{3 / 2}$.

Although the mass of $T$ is significantly below the inflaton mass, there is no Polonyi-like problem [33] associated with $T$. As in the strongly stabilized Polonyi system, the dominant decay mode for $T$ is into a gravitino pair [12], with a decay rate proportional to $m_{3 / 2}^{3} M_{P}^{3} / \Lambda_{T}^{5}$ [34]. The problem of entropy production is easily evaded here. For $\Lambda_{T} \lesssim 10^{-4}$, the modulus decays before the inflaton and plays a little role in subsequent reheating processes. As a result, there is no additional constraint from the overproduction of gravitinos (and ultimately the lightest supersymmetric particle). Since our stabilization term in the Kähler potential should be thought of as an effective interaction, consistency would require $\Lambda_{T}>m_{T}$ or $\Lambda_{T}>(48)^{1 / 4}\left(m_{3 / 2} M_{P}\right)^{1 / 2}$. Thus there is a substantial range of values for $\Lambda_{T}$ for which there is no moduli problem; see [12] for further details. It is interesting that $\Lambda_{T}=M$ lies within the allowed range.

With the modulus fixed at $\langle T\rangle=1 / 2$ and $\operatorname{Im} \phi=0$ (which minimizes the potential), so that $\operatorname{Re} \phi=\phi$, we can write the scalar potential as

$$
\begin{aligned}
V= & 12 a_{1} a_{2}+12 a_{2} M\left(\frac{\phi^{2}}{2}-\frac{\phi^{3}}{3 \sqrt{3}}\right) \\
& +3 M^{2}\left(\frac{\phi}{\sqrt{3}+\phi}\right)^{2} .
\end{aligned}
$$

The first term is the cosmological constant, and the second term is a perturbation of the inflaton potential that has a 
negligible effect on the inflationary dynamics because $a_{2} \ll M$. We then make the following field redefinition to obtain a canonical kinetic term for $\operatorname{Re} \phi$ :

$$
\operatorname{Re} \phi=\sqrt{3} \tanh (x / \sqrt{6}) .
$$

With this field redefinition and dropping terms proportional to $a_{1}$ and $a_{2}$, we obtain the Starobinsky potential along the $\operatorname{Re} \phi$ direction:

$$
V=\frac{3}{4} M^{2}\left(1-e^{-\sqrt{2 / 3} x}\right)^{2} .
$$

The first two terms in Eq. (9) can be written as

$$
\Delta V=\Lambda+6 a_{2} M \tanh ^{2}(x / \sqrt{6})(3-2 \tanh (x / \sqrt{6})),
$$

where we have defined the cosmological constant $\Lambda=12 a_{1} a_{2}$. We note that, at large $x, \Delta V$ adds a relatively small amount $6 a_{2} M$ to the Starobinsky plateau value of $(3 / 4) M^{2}$.

To visualize slow-roll inflation in the $\operatorname{Re} \phi-\operatorname{Re} T$ plane, we use the following field redefinition:

$$
\operatorname{Re} T=\frac{1}{2} e^{-\sqrt{\frac{2}{3} \rho}}
$$

together with the field redefinition $\operatorname{Re} \phi \rightarrow x$ (10). The scalar potential acquires a complicated form in terms of $(x, \rho)$ that we do not display here, which reduces to the form $V+\Delta V$ given by (11) and (12) when $\rho=0$. We assume that the number of $e$-folds until inflation ends is $N=55$, which is realized with the nominal choice of $x=5.35$ and $\rho=0$, yielding the tensor-to-scalar ratio

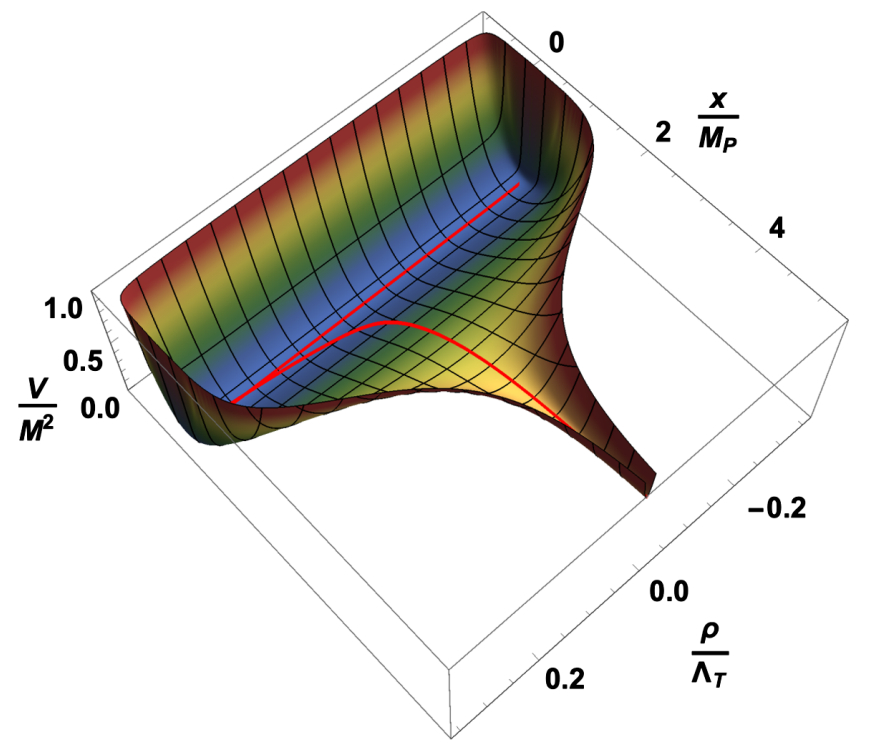

FIG. 1. Realization of the Starobinsky inflationary potential with the initial values of $x(0)=5.35, \rho(0)=0$. The amplitude of the oscillation and the location of the barrier is given by $\left\langle\rho / \Lambda_{T}\right\rangle_{\text {Min }} \sim 0.34$. $r=0.0035$ and the spectral tilt $n_{s}=0.965[35,36]$. The potential in the $x-\rho$ plane is shown in Fig. 1. The field $x$ exits the dS plateau and rolls down towards a potential barrier on the left, located at $x=0, \rho \approx 0.34 \Lambda_{T}$. Then the field $\rho$ evolves slowly towards the global minimum located at $x=0, \rho=0$, and starts spiraling about the minimum with initial amplitude $\langle\rho\rangle_{\text {Min }} \approx 0.34 \Lambda_{T}$ and $\langle x\rangle \approx M_{P}$ until $\rho$ (or $T$ ) decay which occurs well before reheating when the inflaton decays.

\section{STANDARD MODEL INTERACTIONS}

Finally, we consider the full model with SM-like fields characterized by the Kähler function (2), and the full superpotential $W=W_{d S}+W_{I}+W_{\mathrm{SM}}$ (3). After the modulus acquires its vacuum expectation value, $\langle T\rangle=\frac{1}{2}$, and as the inflaton settles to its minimum, the reheating process begins. The coupling $y \phi X^{i} X^{j}$ provides a decay channel for the inflaton and, assuming instantaneous reheating, the reheat temperature is $T_{R} \sim y\left(M M_{P}\right)^{1 / 2}$.

At the minimum, $\langle T\rangle=1 / 2$ and $\langle\phi\rangle=0$, and the potential for the SM-like fields $X^{i}$ can be written as follows in the limit $M_{P} \rightarrow \infty$ :

$V_{\mathrm{SM}}=\sum_{i}\left|W_{X^{i}}\right|^{2}+2 a_{2} \mu\left(X^{i} X^{j}+\right.$ H.c. $)+12 a_{1} a_{2}$,

where $W_{X^{i}} \equiv \partial W / \partial X^{i}$. The first term in (14) is simply a (global) supersymmetric potential term for the Standard Model fields $X^{i}$ in no-scale supergravity. The second term is generated from supersymmetry breaking and appears as an effective supersymmetry-breaking bilinear term, $B_{0}=2 a_{2}$. The third term is, again, our cosmological constant. Gaugino masses can be generated if the gauge kinetic function $f_{\alpha \beta}$ is a function of $T$, so that $m_{1 / 2} \simeq F_{T}\left|f_{T}^{\dagger} / f\right| / 2 \propto m_{3 / 2}$.

As an alternative to (2), one could also consider the case of twisted matter fields, where the kinetic term $X X^{\dagger}$ is taken out of the logarithm in Eq, (2). In such a model, additional soft mass terms are generated as the scalar potential becomes

$$
\begin{aligned}
V_{\mathrm{SM}}= & \sum_{i}\left|W_{X^{i}}\right|^{2}+\left(a_{1}-a_{2}\right)^{2} \sum_{i}\left|X^{i}\right|^{2} \\
& +\left(2 a_{1}+4 a_{2}\right) \mu\left(X^{i} X^{j}+\text { H.c. }\right) \\
& +3\left(a_{1}+a_{2}\right)\left[\lambda X^{i} X^{j} X^{k}+y \phi X^{i} X^{j}+\text { H.c. }\right] \\
& +12 a_{1} a_{2},
\end{aligned}
$$

where we see soft scalar masses with $m_{0}=\left(a_{1}-a_{2}\right)=m_{3 / 2}$, a bilinear term, $B_{0}=\left(2 a_{1}+4 a_{2}\right)$, and trilinear $A$ terms, $A_{0}=3\left(a_{1}+a_{2}\right)$.

\section{SUMMARY}

We have outlined in this paper a simple no-scale supergravity framework for sub-Planckian physics capable of 
including modulus fixing, Starobinsky-like inflation at a scale $\mathcal{O}\left(10^{13}\right) \mathrm{GeV}$, supersymmetry breaking at a scale $\mathcal{O}\left(10^{3}\right) \mathrm{GeV}$, and a small positive cosmological constant (dark energy density). This model should not be regarded as fundamental, but rather as an effective field theory that should, we believe, ultimately be derived from a suitable variant of string theory. In a more complete study of the dynamics of this model, the renormalization-group evolution of the supersymmetry-breaking terms would be able to drive the effective Higgs mass-squared negative, triggering electroweak symmetry breaking [37] and the corresponding change in the vacuum energy density. As mentioned earlier, the parameters $a_{1,2}$ should be chosen such that the dark energy density takes its physical value $\mathcal{O}\left(10^{-120}\right)$ after this contribution is included. Thus the electroweak scale could be generated dynamically in this framework, though we have no new suggestions to offer concerning the origins of the inflationary, supersymmetry-breaking, or dark energy scales. Finally, we note that it would be interesting to explore the extension of this scenario to include grand unification [38] and related issues such as neutrino masses and mixing [25]. However, such topics lie beyond the scope of this paper.

\section{ACKNOWLEDGMENTS}

We would like to thank K. Kaneta for useful discussions. The work of J.E. was supported in part by the United Kingdom STFC Grant No. ST/P000258/1, and in part by the Estonian Research Council via a Mobilitas Pluss grant. The work of D. V.N. was supported in part by the Department of Energy Grant No. DE-FG02-13ER42020 and in part by the Alexander S. Onassis Public Benefit Foundation. The work of K. A. O. was supported in part by Department of Energy Grant No. DE-SC0011842 at the University of Minnesota.
[1] L. Maiani, Conf. Proc. C 7909031, 1 (1979); G. 't Hooft, C. Itzykson, A. Jaffe, H. Lehmann, P. K. Mitter, I. M. Singer, and R. Stora, NATO Sci. Ser. B 59, 1 (1980); E. Witten, Phys. Lett. B 105, 267 (1981).

[2] J. R. Ellis, D. V. Nanopoulos, K. A. Olive, and K. Tamvakis, Phys. Lett. B 118, 335 (1982).

[3] H. P. Nilles, Phys. Rep. 110, 1 (1984).

[4] E. Cremmer, S. Ferrara, C. Kounnas, and D. V. Nanopoulos, Phys. Lett. B 133, 61 (1983).

[5] A. B. Lahanas and D. V. Nanopoulos, Phys. Rep. 145, 1 (1987).

[6] E. Witten, Phys. Lett. 155B, 151 (1985).

[7] A. A. Starobinsky, Phys. Lett. 91B, 99 (1980).

[8] J. Ellis, D. V. Nanopoulos, and K. A. Olive, Phys. Rev. Lett. 111, 111301 (2013).

[9] J. Ellis, B. Nagaraj, D. V. Nanopoulos, and K. A. Olive, J. High Energy Phys. 11 (2018) 110.

[10] J. Polonyi, Budapest preprint KFKI-1977-93.

[11] R. Barbieri, S. Ferrara, and C. A. Savoy, Phys. Lett. 119B, 343 (1982).

[12] J. L. Evans, M. A. G. García, and K. A. Olive, J. Cosmol. Astropart. Phys. 03 (2014) 022.

[13] J. Ellis, M. A. G. García, D. V. Nanopoulos, and K. A. Olive, J. Cosmol. Astropart. Phys. 10 (2015) 003.

[14] M. C. Romão and S. F. King, J. High Energy Phys. 07 (2017) 033.

[15] J. Ellis, D. V. Nanopoulos, K. A. Olive, and S. Verner, J. High Energy Phys. 03 (2019) 099.

[16] S. Kachru, R. Kallosh, A. D. Linde, and S. P. Trivedi, Phys. Rev. D 68, 046005 (2003).

[17] J. R. Ellis, C. Kounnas, and D. V. Nanopoulos, Phys. Lett. 143B, 410 (1984).

[18] J. Ellis, D. V. Nanopoulos, and K. A. Olive, J. Cosmol. Astropart. Phys. 10 (2013) 009.
[19] The part of the model that has $\mathrm{SU}(2,1) / \mathrm{SU}(2) \times \mathrm{U}(1)$ symmetry is found in Eq. (2) when $b=c=0$ and the SM fields are ignored. If the SM fields are included the symmetry is upgraded to an $\mathrm{SU}(\mathrm{N}, 1) / \mathrm{SU}(\mathrm{N}) \times \mathrm{U}(1)$ symmetry.

[20] J. R. Ellis, C. Kounnas, and D. V. Nanopoulos, Nucl. Phys. B241, 406 (1984).

[21] D. Roest and M. Scalisi, Phys. Rev. D 92, 043525 (2015).

[22] A model based on $W=T^{3}-a_{1}+f(\phi)$ was advocated in J. Ellis, D. V. Nanopoulos, and K. A. Olive, Phys. Rev. D 97, 043530 (2018).

[23] Note that the common relation $m_{3 / 2}=F / \sqrt{3}$ is not satisfied here as we do not have a Minkowski vacuum. Recalling that we can write $V=G^{i j} F_{i} F_{j}-3 m_{3 / 2}^{2}$, we easily recover Eq. (7) at the minimum.

[24] We note that the roles and values of $a_{1}$ and $a_{2}$ are largely interchangeable.

[25] J. Ellis, M. A. G. García, N. Nagata, D. V. Nanopoulos, and K. A. Olive, J. Cosmol. Astropart. Phys. 11 (2016) 018; 07 (2017) 006; 04 (2019) 009.

[26] M. Dine, R. Kitano, A. Morisse, and Y. Shirman, Phys. Rev. D 73, 123518 (2006); R. Kitano, Phys. Lett. B 641, 203 (2006); R. Kallosh and A. D. Linde, J. High Energy Phys. 02 (2007) 002; H. Abe, T. Higaki, and T. Kobayashi, Phys. Rev. D 76, 105003 (2007); J. Fan, M. Reece, and L.-T. Wang, J. High Energy Phys. 09 (2011) 126.

[27] E. Dudas, C. Papineau, and S. Pokorski, J. High Energy Phys. 02 (2007) 028; H. Abe, T. Higaki, T. Kobayashi, and Y. Omura, Phys. Rev. D 75, 025019 (2007).

[28] R. Kallosh, A. Linde, K. A. Olive, and T. Rube, Phys. Rev. D 84, 083519 (2011); A. Linde, Y. Mambrini, and K. A. Olive, Phys. Rev. D 85, 066005 (2012).

[29] E. Dudas, A. Linde, Y. Mambrini, A. Mustafayev, and K. A. Olive, Eur. Phys. J. C 73, 2268 (2013). 
[30] J. L. Evans, M. Ibe, K. A. Olive, and T. T. Yanagida, Eur. Phys. J. C 73, 2468 (2013); J. L. Evans, K. A. Olive, M. Ibe, and T. T. Yanagida, Eur. Phys. J. C 73, 2611 (2013).

[31] K. Nakayama, F. Takahashi, and T. T. Yanagida, Phys. Lett. B 718, 526 (2012).

[32] M. A. G. García and K. A. Olive, J. Cosmol. Astropart. Phys. 09 (2013) 007.

[33] G. D. Coughlan, W. Fischler, E. W. Kolb, S. Raby, and G. G. Ross, Phys. Lett. B 131, 59 (1983); A. S. Goncharov, A. D. Linde, and M. I. Vysotsky, Phys. Lett. 147B, 279 (1984); J. Ellis, D. V. Nanopoulos, and M. Quiros, Phys. Lett. B 174, 176 (1986); T. Banks, D. B. Kaplan, and A. E. Nelson, Phys. Rev. D 49, 779 (1994); B. De Carlos, J. A. Casas, F. Quevedo, and E. Roulet, Phys. Lett. B 318, 447 (1993).
[34] Up to a factor of 216, the decay rate for $T$ is the same as the decay rate for a strongly stabilized Polonyi field. The limit on $\Lambda_{T}$ will in general depend on $m_{\phi}, m_{3 / 2}$ and $y$.

[35] N. Aghanim et al. (Planck Collaboration), arXiv:1807 .06209; Y. Akrami et al. (Planck Collaboration), Astrophys. Space Sci. 364, 69 (2019).

[36] Although during the later stages of inflation the dynamics of $T$ and $\phi$ are complicated by kinetic mixing, the effects discussed in J. Ellis, M. A. G. García, D. V. Nanopoulos, and K. A. Olive, J. Cosmol. Astropart. Phys. 01 (2015) 010, hardly alter the predictions for the perturbation spectrum.

[37] J. R. Ellis, A. B. Lahanas, D. V. Nanopoulos, and K. Tamvakis, Phys. Lett. 134B, 429 (1984).

[38] J. R. Ellis, C. Kounnas, and D. V. Nanopoulos, Nucl. Phys. B247, 373 (1984). 